\title{
Magnetic particle imaging: kinetics of the intravascular signal in vivo
}

\author{
This article was published in the following Dove Press journal: \\ International Journal of Nanomedicine \\ 3 September 2014 \\ Number of times this article has been viewed
}

\author{
Julian Haegele' \\ Robert L Duschka' \\ Matthias Graeser ${ }^{2}$ \\ Catharina Schaecke' \\ Nikolaos Panagiotopoulos \\ Kerstin Lüdtke-Buzug ${ }^{2}$ \\ Thorsten M Buzug 2 \\ Jörg Barkhausen' \\ Florian M Vogt' \\ 'Clinic for Radiology and Nuclear \\ Medicine, ${ }^{2}$ Institute of Medical \\ Engineering, University Hospital \\ Schleswig Holstein, Lübeck, Schleswig- \\ Holstein, Germany
}

Background: Magnetic particle imaging (MPI) uses magnetic fields to visualize superparamagnetic iron oxide nanoparticles (SPIO). Today, Resovist ${ }^{\circledR}$ is still the reference SPIO for MPI. The objective of this study was to evaluate the in vivo blood half-life of two different types of Resovist (one from Bayer Pharma AG, and one from I'rom Pharmaceutical Co Ltd) in MPI

Methods: A Resovist concentration of $50 \mu \mathrm{mol} / \mathrm{kg}$ was injected into the ear artery of ten New Zealand White rabbits. Five animals received Resovist distributed by I'rom Pharmaceutical Co Ltd and five received Resovist by Bayer Pharma AG. Blood samples were drawn before and directly after injection of Resovist, at 5, 10, and 15 minutes, and then every 15 minutes until 120 minutes after the injection. The MPI signal of the blood samples was evaluated using magnetic particle spectroscopy.

Results: The average decline of the blood MPI signal from the two distributions differed significantly $(P=0.0056)$. Resovist distributed by Bayer Pharma AG showed a slower decline of the MPI signal (39.7\% after 5 minutes, $20.5 \%$ after 10 minutes, and $12.1 \%$ after 15 minutes) compared with Resovist produced by I'rom Pharmaceutical Co Ltd (20.4\% after 5 minutes, $7.8 \%$ after 10 minutes, no signal above noise level after 15 minutes).

Conclusion: In MPI, the blood half-life of an SPIO tracer cannot be equalized to the blood half-life of its MPI signal. Resovist shows a very rapid decline of blood MPI signal and is thus not suitable as a long circulating tracer. For cardiovascular applications in MPI, it may be used as a bolus tracer.

Keywords: magnetic particle imaging, superparamagnetic iron oxide nanoparticles, magnetic particle spectroscopy, blood half-life time, Resovist ${ }^{\circledR}$

\section{Introduction}

Magnetic particle imaging (MPI) is a new three-dimensional imaging method using static and dynamic magnetic fields to visualize the distribution of superparamagnetic iron oxide nanoparticles (SPIOs). ${ }^{1}$ Imaging relies thereby on three characteristics of the SPIO, ie; their superparamagnetic nature, their nonlinear magnetization curve, and their ability to be magnetically saturated. ${ }^{2}$ Because of these characteristics, MPI can visualize and quantify the spatial distribution of SPIO, providing very high temporal and spatial resolution combined with high signal-to-noise ratios. Possible application scenarios for MPI might include cardiovascular imaging and even guidance of cardiovascular interventions. ${ }^{3,4}$ In principle, MPI would allow visualization and quantification of vascular pathologies while also being able to determine additional functional parameters in one examination. Further, MPI could be used as an imaging method for percutaneous transluminal coronary angioplasty, if indicated.

Apart from the need for suitable MPI scanners, this diagnostic and therapeutic pathway relies on appropriate tracer material. Ideally, long circulating SPIO are desirable, 
and should be able to provide a sufficient signal within the vessels after a single tracer injection at the beginning of the procedure, so a diagnostic scan and intervention can be done consecutively. The circulation time of SPIO in blood is mainly determined by their overall so-called hydrodynamic diameter. Although SPIO with a greater dimension of the iron oxide core, and consequently a larger hydrodynamic particle size, improve the superparamagnetic behavior of contrast agents in general, the coatings play a major role in the biodistribution and lesional uptake of SPIO. Previous reports demonstrate that SPIO with a hydrodynamic size greater than $50 \mathrm{~nm}$ have in common rapid and specific uptake by the reticuloendothelial system, and thus are cleared rapidly from the blood stream. Compared with magnetic resonance imaging (MRI), the properties of SPIO not only influence pharmacokinetics but also greatly influence the quality and sensitivity of the entire MPI system. To achieve high performance in MPI, an ideal tracer should exhibit SPIO with a homogeneously distributed iron core diameter of $30 \mathrm{~nm}$, resulting in a relatively high hydrodynamic diameter and a shorter half-life in blood. ${ }^{1}$

Although the characteristics of an ideal MPI tracer are well understood in theory, and this issue has been addressed by many working groups, no MPI-specific tracer has reached the marketplace so far. Since the advent of MPI, many SPIO agents have been evaluated for their usefulness in MPI. ${ }^{5}$ Resovist $^{\circledR}$, an approved T2* contrast agent for MRI, is the most important benchmark material, and remains the best performing commercial SPIO for MPI today and the standard of reference for evaluation of MPI hardware and application, although it has known drawbacks, ie, a hydrodynamic diameter greater than $50 \mathrm{~nm}$ and therefore, a limited in vivo distribution. ${ }^{6}$ However, in the context of cardiovascular MPI, knowledge about the pharmacokinetics of Resovist, especially its half-life in blood, is important. Naturally, this issue was investigated about 20 years ago, when Resovist was evaluated as a contrast agent for MRI. ${ }^{7}$ However, because the imaging principle is fundamentally different in MPI, results from MRI cannot be easily extrapolated to MPI, as not all Resovist particles contribute equally to the MPI signal and larger particles are cleared more rapidly by the reticuloendothelial system (RES). Thus, the aim of this study was to evaluate the blood half-life of MPI using magnetic particle spectroscopy (MPS), given that it may differ significantly from the blood half-life in MRI. Marketing of Resovist by Bayer Pharma AG (Berlin, Germany) has been discontinued, and it is only available now from the Japanese distributor, I'rom Pharmaceutical Co Ltd (Tokyo, Japan). However, until now, most MPI experiments were done using Resovist from Bayer Pharma AG, whereas most working groups now use Resovist from I'rom Pharmaceutical Co Ltd.

\section{Materials and methods}

The experimental protocol was approved by the state regulations governing the performance of animal studies. All experiments were conducted under strict adherence to the guidelines for the care and use of laboratory animals as described by the National Institutes of Health.

\section{Iron oxide nanoparticles}

Two different versions of Resovist were studied, ie, that distributed by Bayer Pharma AG in Europe and that distributed by I'rom Pharmaceutical Co Ltd. Resovist was developed as a RES-specific MRI contrast agent for liver imaging. It was approved for clinical use in 2001 in Europe and in 2002 in Japan. ${ }^{8,9}$ Marketing of the Bayer Pharma AG product has now been discontinued in Europe.

\section{In vivo study}

Resovist ( $50 \mu \mathrm{mol} / \mathrm{kg}$ ) was administered into the ear artery of ten female New Zealand White rabbits after drawing of a control blood sample. Five animals received Resovist by I'rom Pharmaceutical Co Ltd and five by Bayer Pharma AG, respectively. Each rabbit weighed approximately $4 \mathrm{~kg}$. Directly (about 30 seconds) after administration of Resovist, a second blood sample was collected as a baseline sample $\left(\mathrm{T}_{0}\right)$ from the contralateral ear artery to avoid contamination with residual Resovist. This was repeated after 5, 10, 15, 30, 45, 60, 75, 90, 105, and 120 minutes. During the experiments, the animals were kept under full anesthesia by bodyweight-adapted subcutaneous injection of ketamine hydrochloride $(0.7 \mathrm{~mL} / \mathrm{kg}$ bodyweight; Ceva Animal Health, Düsseldorf, Germany) and xylazine hydrochloride (0.3 mL/kg bodyweight; Ceva Animal Health). Blood samples were collected in accordance with the recommendations of the German Society for Laboratory Animal Science. ${ }^{10}$

\section{Magnetic particle spectroscopy}

The MPI signal was evaluated using MPS. MPS can be described as a zero-dimensional system that measures the remagnetization spectrum of SPIO. In MPS, only the oscillating magnetic field that excites the SPIO is used. The magnetic gradient field, which is used for spatial encoding in MPI, is omitted. ${ }^{11}$ Using MPS it is possible to evaluate the quality of the MPI signal of particles, so the applicability 
of the particles for MPI can be characterized. In an ideal superparamagnetic particle, the spectra consist only of odd harmonics due to the symmetry of the particle magnetization curve. In an experimental setup, even harmonics are added by the earth's magnetic field, but can be neglected in evaluation of the particles. ${ }^{12}$ Thus, the signal strength can be determined by evaluating the odd harmonics of SPIO above noise level. The magnitude of the signal is proportional to the concentration of the SPIO.

As in previous experiments, ${ }^{5}$ the MPS excitation frequency was $25 \mathrm{kHz}$ (as is most common in MPI systems), the magnetic field amplitude was $20 \mathrm{mT}$, and the acquisition time was 5 seconds (corresponding to 125,000 repetitions) to provide a high signal-to-noise ratio.

\section{Ex vivo blood sample analysis}

Ten milliliters of each blood sample were transferred into individual Eppendorf tubes directly after sampling, and then measured using MPS. The signal strength of the odd harmonics from the third to the 49th harmonic was measured at each time point and related to the signal strength of the baseline sample $\left(\mathrm{T}_{0}\right)$. Thus, the decline of the MPI signal in blood could be described in relation to the signal maximum at baseline directly after administration of Resovist. For each group, ie, Resovist distributed by Bayer Pharma AG and I'rom Pharmaceutical Co Ltd, respectively, the average ( \pm standard deviation) decline in signal strength was calculated for each time point.

\section{Evaluation of Resovist}

Ten microliters of pure Resovist $(500 \mu \mathrm{mol} \mathrm{Fe} / \mathrm{mL})$ were drawn from each package unit and diluted using Ringer's solution to $400,300,200,100,80,60,40,20,10,8,6,4,2$, and $1 \mu \mathrm{mol} \mathrm{Fe} / \mathrm{mL}$. All samples were measured using MPS. The hydrodynamic diameter and size distribution of the SPIO for the two different types of Resovist were measured using photon cross correlation spectroscopy (Nanophox, Sympatec $\mathrm{GmbH}$, Clausthal-Zellerfeld, Germany). In photon cross correlation spectroscopy, the particle size is measured by three-dimensional cross correlation of the scattered light of two intersecting focused laser beams. ${ }^{13}$

\section{Statistical analysis}

The statistical significance of the difference in the signal decay in blood between the two types of Resovist was calculated using the Mann-Whitney $U$-test. Further, the statistical significance of the difference between the two package units in the dilution series was calculated in the same way. In both cases, $P$-values $<0.05$ were considered to be statistically significant.

\section{Results}

Injection of contrast medium and collection of blood samples proved feasible. All animals survived the experiments without any notable adverse reactions to the contrast agents.

The average MPI signal in blood declined to $30 \%$ of the baseline signal 5 minutes after injection. Ten and 15 minutes after injection, the average MPI signal was $14.8 \%$ and $7 \%$, respectively, of the baseline signal. Within 30 minutes, the MPI signal was below the noise level of the MPS (see Table 1). Regardless of this result, the MPI signal was measured consecutively until 120 minutes after application; however, it was still not possible to detect any signal above the noise level. The noise level was as high as $3.4 \%$ of the average baseline signal.

For further evaluation, the signal decline for both types of Resovist was compared. The signal for Resovist from Bayer Pharma AG decreased to $39.7 \%$ of baseline after 5 minutes, to $20.5 \%$ after 10 minutes, and to $12.1 \%$ after 15 minutes. The signal for Resovist from I'rom Pharmaceutical Co Ltd showed a rapid decrease to $20.4 \%$ of the baseline signal after 5 minutes and to $7.8 \%$ after 10 minutes (see Table 1 and Figure 1); in contrast with Resovist from Bayer Pharma AG, the signal dropped below noise level even after 15 minutes. This difference was statistically significant (Mann-Whitney $U$-test, $P=0.0056$ ).

Table I Strength of MPI signal in blood, measured by MPS as the percentage of the highest blood MPI signal directly after administration of Resovist ${ }^{\circledR}$ at baseline

\begin{tabular}{|c|c|c|c|c|c|}
\hline & Baseline & 5 minutes & 10 minutes & I 5 minutes & 30 minutes \\
\hline Resovist (Bayer Pharma AG) & $100 \%$ & $39.7 \% \pm 8 \%$ & $20.5 \% \pm 6 \%$ & $12.1 \% \pm 5 \%$ & $31 \% \pm 3 \%$ \\
\hline Resovist (l'rom Pharmaceutical Co Ltd) & $100 \%$ & $20.4 \% \pm 10 \%$ & $7.8 \% \pm 4 \%$ & $1.9 \% \pm 2 \%{ }^{\mathrm{a}}$ & $0.7 \% \pm 0.4 \%^{a}$ \\
\hline Mean & $100 \%$ & $30.0 \% \pm 13 \%$ & $14.8 \% \pm 7.8 \%$ & $7.0 \% \pm 6 \%$ & $1.9 \% \pm 2 \% \mathrm{a}$ \\
\hline
\end{tabular}

Notes: Values are shown as the mean \pm standard deviation. There is a statistically significant difference in signal decay between of the two types of Resovist $(P=0.0056$, Mann-Whitney U-test). From 30 to 120 minutes, in case of Resovist (l'rom Pharmaceutical Co Ltd) after 15 minutes, there was no measurable signal above the noise level of the magnetic particle spectrometer. ${ }^{a}$ Signal below noise level.

Abbreviations: MPI, magnetic particle imaging; MPS, magnetic particle spectroscopy. 


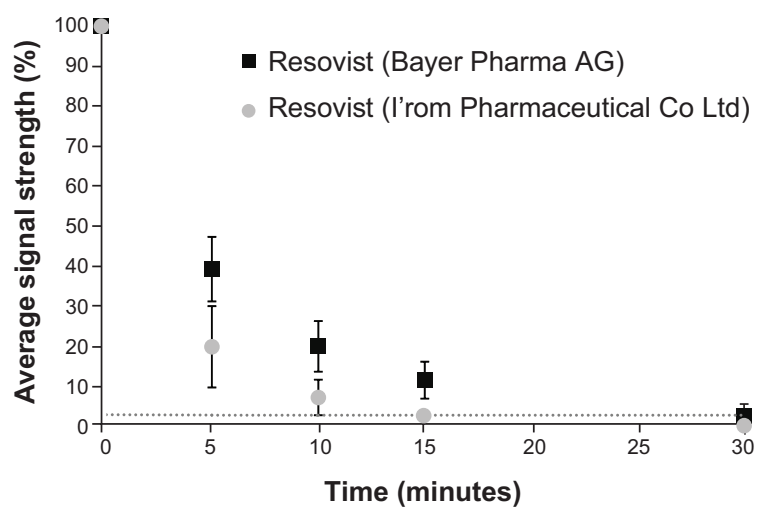

Figure I Average strength of the MPI signal in blood as a percentage of the highest blood MPI signal directly after administration of Resovist ${ }^{\circledR}$ (time, 0 minutes).

Notes: The noise level of the MPS system is indicated by the dotted line. The MPI signal of Resovist from l'rom Pharmaceutical Co Ltd decays significantly more rapidly than that of Resovist from Bayer Pharma AG, and drops below the noise level after 15 minutes.

Abbreviations: MPI, magnetic particle imaging; MPS, magnetic particle spectroscopy.

In contrast with the signal decay, the absolute signal strength of the baseline blood MPI signal was not significantly different between the two Resovist products (1.60e$8 \pm 8,09 \mathrm{e}-9 \mathrm{Am}^{2} \mathrm{~Hz}^{-1}$ for Resovist from Bayer Pharma AG and $\mathrm{J} 1.98 \mathrm{e}-8 \pm 4,13 \mathrm{e}-9 \mathrm{Am}^{2} \mathrm{~Hz}^{-1}$ for Resovist from I'rom Pharmaceutical Co Ltd, $P=0.3953$, Mann-Whitney $U$-test, see Figure 2).

In addition, the dilution series showed no statistical significant difference in MPI signal decay between the two compounds (Mann-Whitney $U$-test, $P=0.6241$, see Figure 3 ). Photon cross correlation spectroscopy showed a median hydrodynamic diameter $\left(\mathrm{x}_{50}\right)$ of $70.54 \mathrm{~nm}$ for Resovist from Bayer Pharma AG and $61.86 \mathrm{~nm}$ for Resovist from I'rom Pharmaceutical Co Ltd. The size distribution was

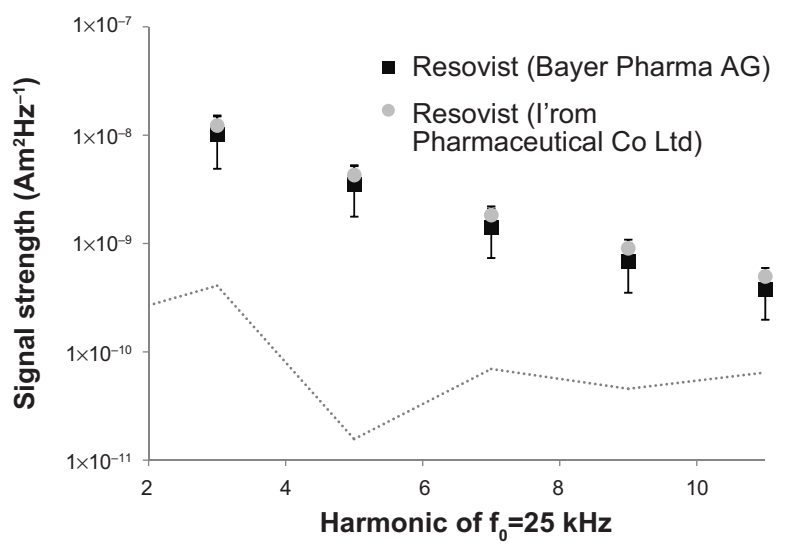

Figure 2 Average baseline signal strength of Resovist ${ }^{\circledast}$ from Bayer Pharma AG and from I'rom Pharmaceutical Co Ltd.

Notes: The first five odd harmonics of the excitation frequency $f_{0}=25 \mathrm{kHz}$ are shown. There is no statistically significant difference in signal strength at baseline, indicating a similar performance of both products in MPI considering the MPI signal. The noise level of the MPS system is indicated by the dotted gray line.

Abbreviations: MPI, magnetic particle imaging; MPS, magnetic particle spectroscopy.

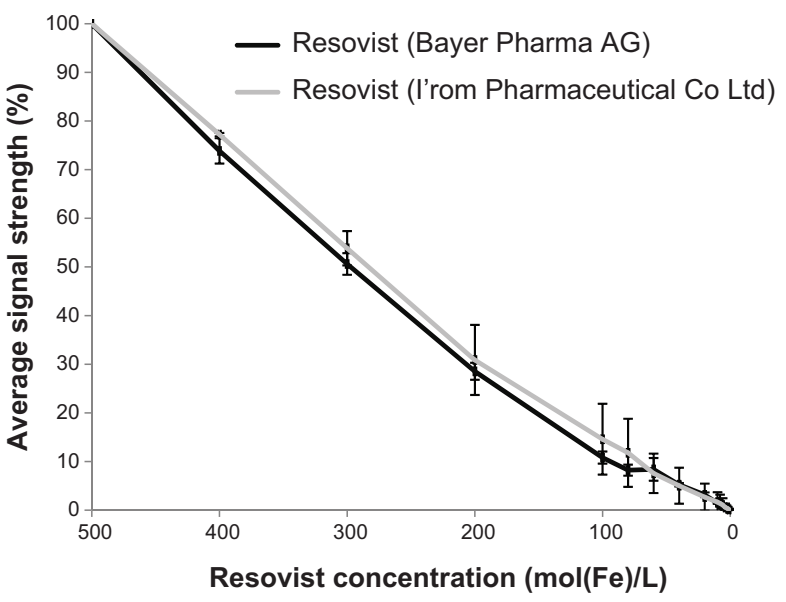

Figure 3 Average signal strength of a dilution series of Resovist ${ }^{\circledR}$ from Bayer Pharma AG and from l'rom Pharmaceutical Co Ltd.

Notes: The signal strength was measured using MPS. The performance of Resovist from l'rom Pharmaceutical Co Ltd is slightly but not significantly better at higher concentrations. At lower concentrations, no difference can be detected between the two types of Resovist.

Abbreviation: MPS, magnetic particle spectroscopy.

$\mathrm{x}_{10} 60.41 \mathrm{~nm}$ and $\mathrm{x}_{90} 82.07 \mathrm{~nm}\left(\mathrm{x}_{10} / \mathrm{x}_{90}=1.36\right)$ for Resovist from Bayer Pharma AG and $x_{10}$ and $x_{90} 51.16 \mathrm{~nm}$ and $73.08 \mathrm{~nm}\left(\mathrm{x}_{10} / \mathrm{x}_{90}=1.4\right)$ for Resovist from I'rom Pharmaceutical Co Ltd.

\section{Discussion}

This study carries three messages that we believe to be important. First, Resovist is not suitable as an intravascular tracer in terms of prolonged circulation. Given the current lack of alternatives in MPI, it may be used as a bolus tracer either intravenously for a diagnostic scan or be applied during a cardiovascular intervention using an intravascular catheter. This of course is limited by the maximum permissible dose. The present study underlines once more the need for a dedicated MPI tracer with a prolonged blood half-life of its MPI signal. Second, it highlights the fact that the blood half-life of the MPI tracer cannot be equalized to the blood half-life time of the MPI signal. The concordance of these two values depends on the fraction of particles contributing to the MPI signal. Third, the difference in blood half-life between Resovist sourced from Bayer Pharma AG and that from I'rom Pharmaceutical Co Ltd has to be taken into account when comparing different studies. Further, due to the inhomogeneous size distribution from batch to batch, it is even possible that tracers of the same type show differences in blood half-life.

The rapid decay of the MPI signal is in concordance with the initial phase of elimination of Resovist in the studies conducted in humans using MRI. In their MRI studies, Hamm et $\mathrm{al}^{14}$ observed two phases of elimination of the contrast 
agent. In the first phase, about $80 \%$ of the Resovist concentration was cleared, with a blood half-life of 3.9-5.8 minutes, whereas that of the second phase was 2.4-3.6 hours. The blood half-life was therefore independent of the concentration administered. ${ }^{14}$ The package insert for Resovist states that the first phase is $15.6 \pm 11.4$ minutes and the second phase is $4.36 \pm 0.75$ hours. $^{8}$ In contrast, no second phase of tracer elimination could be detected using MPS in our study. This difference can be explained by the different principles of signal generation in MPI and MRI, and the broad size distribution of the SPIO in Resovist. In MRI, the effect of the SPIO is a shortening of the T2 time in the surrounding tissues, leading to a signal loss, especially in T2* weighted images. Thus, in MRI, SPIO work as an indirect contrast agent influencing the signal characteristics of the surrounding tissue. This occurs independently of the size of the SPIO, as long as they still have superparamagnetic characteristics.

In MPI, the SPIO are visualized directly by their change of magnetization in an oscillating magnetic field. Thus, they work like a tracer and not like a contrast agent. As mentioned above, the diameter of the iron core should be around $30 \mathrm{~nm}$ for an ideal MPI signal. ${ }^{1}$ In Resovist, theoretically only 3\% of particles meet this criterion and contribute substantially to the MPI signal. ${ }^{1}$ Recently, Eberbeck et $\mathrm{al}^{6}$ demonstrated by analysis of the $\mathrm{M}(\mathrm{H})$ curve for Resovist that there are clusters of smaller SPIO in Resovist that behave like single domain particles and have an effective mean iron core diameter of $24 \pm 1 \mathrm{~nm}$. In their data, this fraction of particle clusters amounted to $30 \% \pm 3 \%$ of the nanoparticles in Resovist and explain its good MPI performance. ${ }^{6}$ The other fraction of SPIO in Resovist has an effective mean iron core diameter of $5.5 \pm 3 \mathrm{~nm}$, and does not contribute substantially to the MPI signal. ${ }^{6}$ It is important to note that the physical mean iron core diameter as measured by, eg, transmission electron microscopy and vibrating sample magnetometry, is between $5 \mathrm{~nm}$ and $15 \mathrm{~nm}$, as described by many working groups, and the clusters contributing most of Resovist's MPI-signal as described in Eberbeck et $\mathrm{al}^{6}$ contain these smaller particles, as can also be shown by transmission electron microscopy. ${ }^{6,15-19}$ These clusters may also account for a fraction with a larger hydrodynamic diameter than the other particle fractions in Resovist. These would be cleared more rapidly from the blood by the reticuloendothelial system and the MPI signal would decrease more rapidly than the actual concentration of the tracer measured, eg, on MRI. This explanation is supported by a study reported by Khandhar et al. ${ }^{19}$ The authors evaluated Resovist and a self-designed tracer for their MPI performance in mice and showed that the decay of the MPI signal from Resovist in blood was considerably faster than the decay of the magnetization curve measured by vibrating sample magnetometry. This also indicates that there are Resovist particles left in the blood that do not contribute to the MPI signal. ${ }^{19}$ In contrast with our results, they observed an even faster decrease in the MPI signal to below noise level within 10 minutes. This is probably due to the differing sensitivities of the respective MPS systems, as there are no international standardized systems on the market as yet. Given that Khandhar et $\mathrm{a}^{19}$ did not reveal the dose of Resovist related to body weight of the mice, differences in the dose of Resovist initially applied might explain the shorter "time above noise level" as well. Further, Khandhar et al described an initial slow increase of MPS signal from 0 to 5 minutes after administration. ${ }^{19}$ This also seems to be in contrast with the findings of Weizenecker et al, ${ }^{20}$ who were the first group to present in vivo MPI images, which visualized the beating heart of a mouse after application of a bolus of Resovist from Bayer Pharma AG. An approximate equilibrium was achieved in their study about 15 seconds after intravenous injection, and a rapid decay of the tracer concentration determined by the MPI demonstrator in the vena cava from about $3.8 \mathrm{mmol}$ $\mathrm{Fe} / \mathrm{L}$ at first pass to $0.28 \mathrm{mmol} \mathrm{Fe} / \mathrm{L}$ at third pass 10.2 seconds later was described. ${ }^{20}$ In summary, it seems that the Resovist particles contributing most to the MPI signal are cleared most rapidly from the blood.

Interestingly, we found a significant difference in blood half-life between Resovist manufactured by Bayer Pharma $\mathrm{AG}$ and that manufactured by I'rom Pharmaceutical Co Ltd. However, our measurements do not indicate significant differences in hydrodynamic diameter or size distribution, which would be the most likely explanation for these differences in blood half-life. Resovist from Bayer Pharma AG showed a slightly larger hydrodynamic diameter than that from I'rom Pharmaceutical Co Ltd. A difference in formulation of the polymeric coating is improbable, given that the Japanese company that developed Resovist in cooperation with Schering AG (Berlin, Germany) still produces the drug substance for Resovist, ie, Ferucarbotran (Meito Sangyo Co Ltd, Nagoya, Japan). Further, there is no discrepancy in the baseline signal strength or signal loss in the dilution series between the two types of Resovist, ie, the MPI performance of the tracers. Resovist from I'rom Pharmaceutical Co Ltd performed slightly, albeit not significantly, better than Resovist from Bayer Pharma AG (Figure 3). All MPI-specific research to date regarding particle characterization and dynamics has been conducted using Resovist from Bayer Pharma AG, and there are no such data for Resovist from I'rom Pharmaceutical Co Ltd. Tsuchiya et $\mathrm{al}^{21}$ indicate that the iron core 
diameter of Resovist from I'rom Pharmaceutical Co Ltd is $5 \mathrm{~nm}$, but do not provide a reference for this or a method for how the iron core diameter was measured. Given that the MPI performance of Resovist sourced from the two distributors is almost the same, it is highly probable that the mechanism of clustering of Resovist particles as described by Eberbeck et $\mathrm{al}^{6}$ for Resovist by Bayer Pharma AG holds true, otherwise, the MPI performance would be well below that of Resovist distributed by Bayer Pharma AG.

Thus, for Resovist to be used further in the setting of MPI, two issues have to be considered. Firstly, the distribution of Resovist by Bayer Pharma AG in Europe has been discontinued and it is no longer commercially available. Therefore, Resovist distributed by I'rom Pharmaceutical Co Ltd may be the standard of reference until a dedicated SPIO tracer is marketed, whereas the first in vivo experiments in MPI were conducted using Resovist distributed by Bayer Pharma AG. ${ }^{20}$ This should be considered when evaluating experimental results. Secondly, the difference in blood half-life between the two compounds is significant, but not as high as to indicate a change of application. As we showed, in accordance with other investigators, the MPI signal of Resovist in blood lacks the second, slower elimination phase observed in MRI and rapidly decays below noise level, so is not ideal for applications requiring long circulating or even blood pool tracers. In this study, the MPI signal was measured using MPS, which is known to be the most sensitive method for signal detection; thus, the time frame in which Resovist can be visualized with current and upcoming MPI scanners will probably be even shorter, and Resovist will most likely be suitable only as a bolus tracer for vascular applications.

In addition to the challenges concerning scanner design, the design of tracers will be a key aspect in future development of MPI. SPIO formulations originally designed for MRI do not exhibit ideal MPI characteristics, so research is now focused on development of a dedicated MPI tracer, ie, an SPIO tracer with a homogenously distributed iron core diameter of about $30 \mathrm{~nm} .^{6,18,19,22}$ Here, Resovist serves as an international standard of reference for the performance of MPI using new tracers. MPS is the most important tool for evaluation, but magnetorelaxometry, vibrating sample magnetometry, and $\mathrm{M}(\mathrm{H})$ curves are also important tools, particularly for description, and explain the sometimes complex MPI performance of SPIO. ${ }^{6,18,19}$ Apart from the need for ideal MPI characteristics, mostly determined by the iron core and the homogenous size distribution of the particles, pharmacokinetics have a crucial role in the use of the tracer in vivo. For long circulating tracers, a small hydrodynamic diameter is desirable. Because the iron core diameter should be $30 \mathrm{~nm}$, the minimum hydrodynamic diameter is limited, even if the coating is made of, eg, thin monomer layers. Thus, other strategies also have to be evaluated. Recently, Rahmer et al reported a proof of principle study in which red blood cells in mice were loaded with Resovist, enabling MPI of the vascular system for several hours after application. ${ }^{23}$ Another option might be to modify the coating and charge on the SPIO in order to enhance circulation time. ${ }^{24}$ Outside the MPI community, a lot of research has already been conducted with regard to different SPIO particle coatings and surface modifications for specific areas of application, eg, for targeted imaging or to prolong the blood circulation time, and MPI can benefit from this knowledge. In order to enable cooperation and dissemination of knowledge, a standardized physicochemical testing protocol as a part of particle development is desirable, as already proposed. ${ }^{25,26}$ These standards may even simplify the long way from "bench to bedside".

However, Resovist remains the only industrially produced (in Japan) and approved SPIO formulation applicable for MPI. Feraheme ${ }^{\circledR}$ (ferumoxytol, AMAG Pharmaceuticals, Waltham, MA, USA) is a SPIO formulation approved by the US Food and Drug Administration for the treatment of iron deficiency anemia in adult patients with chronic kidney disease, but is not approved for MPI due to its inadequate MPI signal characteristics.

A limitation of our study is the relatively small number of animals included. Additionally, the diverging performance of the two types of Resovist could not be fully elucidated. Therefore, studies of different batches of Resovist in larger groups of animals may be necessary. Further, analysis of Resovist distributed by I'rom Pharmaceutical Co Ltd corresponding to, ie, Eberbeck et al, should be executed in the future. ${ }^{6}$ Ideally, the pharmacological formulation of the two distributions should be analyzed in detail, because this may be a reason for the different half-life times. Finally, the results need to be confirmed by visualization of vessels in larger animals in real time to determine the effect on MPI in vivo.

\section{Conclusion}

In conclusion, our study shows that Resovist is not a suitable tracer for applications requiring a long circulation time of the MPI tracer in blood. More importantly, our study highlights a difference in a tracer's blood half-life with regard to the MPI signal on the one hand, and the MRI signal and iron concentration on the other. Interestingly, there are even distinctions between different distributions of the same pharmaceutical 
agent, in this case Resovist by Bayer Pharma AG and I'rom Pharmaceutical Co Ltd. Consequentially, all SPIO formulations have to be evaluated for the half-life of the MPI signal in blood before use in MPI.

\section{Acknowledgments}

This work was funded inhouse by the University of Lübeck within the context of the support program "SPP Bildgebung bei Krankheitsprozessen”. The Institute of Medical Engineering and the Clinic for Radiology and Nuclear Medicine are currently receiving a grant from the German Federal Ministry of Education and Research in the context of the cooperation project "Magnetic Particle Imaging Technologie" (Project ID 13N11090).

\section{Disclosure}

The authors report no conflicts of interest in this work.

\section{References}

1. Gleich B, Weizenecker J. Tomographic imaging using the nonlinear response of magnetic particles. Nature. 2005;435(7046):1214-1217.

2. Borgert J, Schmidt JD, Schmale I, et al. Fundamentals and applications of magnetic particle imaging. J Cardiovasc Comput Tomogr. 2012;6(3): $149-153$.

3. Haegele J, Rahmer J, Gleich B, et al. Magnetic particle imaging: visualization of instruments for cardiovascular intervention. Radiology. 2012;265(3):933-938.

4. Goodwill PW, Saritas EU, Croft LR, et al. X-space MPI: magnetic nanoparticles for safe medical imaging. Adv Mater. 2012;24(28): 3870-3877.

5. Ludtke-Buzug K, Haegele J, Biederer S, et al. Comparison of commercial iron oxide-based MRI contrast agents with synthesized high-performance MPI tracers. Biomed Tech (Berl). 2013;58(6):527-533.

6. Eberbeck D, Wiekhorst F, Wagner S, Trahms L. How the size distribution of magnetic nanoparticles determines their magnetic particle imaging performance. Appl Phys Lett. 2011;98(18):182502.

7. Hamm B, Staks T, Taupitz M. SHU 555A. A new superparamagnetic iron oxide contrast agent for magnetic resonance imaging. Invest Radiol. 1994;29 Suppl 2:S87-S89.

8. Bayer Schering Pharma AG. Resovist [package insert]. Leverkusen, Germany: Bayer Schering Pharma AG; 2007.

9. Reimer P, Balzer T. Ferucarbotran (Resovist): a new clinically approved RES-specific contrast agent for contrast-enhanced MRI of the liver: properties, clinical development, and applications. European Radiology. 2003;13(6):1266-1276.
10. Nicklas W. [Society for Laboratory Animal Science]. 2009; http:// www.gv-solas.de/assets/files/PDFs/pdf_PUBLIKATION/tie_ blutentnahme09.pdf. Accessed December 18, 2013. German.

11. Biederer S, Knopp T, Sattel TF, et al. Magnetization response spectroscopy of superparamagnetic nanoparticles for magnetic particle imaging. J Phys D Appl Phys. 2009;42(20):205007.

12. Weaver JB, Rauwerdink AM, Sullivan CR, Baker I. Frequency distribution of the nanoparticle magnetization in the presence of a static as well as a harmonic magnetic field. Med Phys. 2008;35(5):1988-1994.

13. Yang H, Zheng G, Zhang RJ, Dai SG, Mu PA. Development of photon cross-correlation spectroscopy for the characterization of nanoparticles in concentrated suspension. Lasers in Engineering. 2012; 23(5-6):367-381.

14. Hamm B, Staks T, Taupitz M, et al. Contrast-enhanced MR imaging of liver and spleen: first experience in humans with a new superparamagnetic iron oxide. J Magn Reson Imaging. 1994;4(5):659-668.

15. Chen DX, Sun N, Gu HC. Size analysis of carboxydextran coated superparamagnetic iron oxide particles used as contrast agents of magnetic resonance imaging. J Appl Phys. 2009; 106:063906-063909.

16. Ferguson RM, Minard KR, Krishnan KM. Optimization of nanoparticle core size for magnetic particle imaging. J Magn Magn Mater. 2009; 321(10):1548-1551.

17. Ferguson RM, Minard KR, Khandhar AP, Krishnan KM. Optimizing magnetite nanoparticles for mass sensitivity in magnetic particle imaging. Med Phys. 2011;38(3):1619-1626.

18. Ferguson RM, Khandhar AP, Krishnan KM. Tracer design for magnetic particle imaging (invited). J Appl Phys. 2012;111(7): 7B318-317B3185.

19. Khandhar AP, Ferguson RM, Arami H, Krishnan KM. Monodisperse magnetite nanoparticle tracers for in vivo magnetic particle imaging. Biomaterials. 2013;34(15):3837-3845.

20. Weizenecker J, Gleich B, Rahmer J, Dahnke H, Borgert J. Threedimensional real-time in vivo magnetic particle imaging. Phys Med Biol. 2009;54(5):L1-L10.

21. Tsuchiya K, Nitta N, Sonoda A, et al. Histological study of the biodynamics of iron oxide nanoparticles with different diameters. Int $J$ Nanomedicine. 2011;6:1587-1594.

22. Ludwig F, Wawrzik T, Yoshida T, et al. Optimization of magnetic nanoparticles for magnetic particle imaging. IEEE Trans Magn. 2012; 48(11):3780-3783.

23. Rahmer J, Antonelli A, Sfara C, et al. Nanoparticle encapsulation in red blood cells enables blood-pool magnetic particle imaging hours after injection. Phys Med Biol. 2013;58(12):3965-3977.

24. Almeida JP, Chen AL, Foster A, Drezek R. In vivo biodistribution of nanoparticles. Nanomedicine (Lond). 2011;6(5):815-835.

25. Dufort S, Sancey L, Coll JL. Physico-chemical parameters that govern nanoparticles fate also dictate rules for their molecular evolution. $A d v$ Drug Deliv Rev. 2012;64(2):179-189.

26. Szekeres M, Tóth I, Illés E, et al. Chemical and colloidal stability of carboxylated core-shell magnetite nanoparticles designed for biomedical applications. Int J Mol Sci. 2013;14(7):14550-14574.
International Journal of Nanomedicine

\section{Publish your work in this journal}

The International Journal of Nanomedicine is an international, peerreviewed journal focusing on the application of nanotechnology in diagnostics, therapeutics, and drug delivery systems throughout the biomedical field. This journal is indexed on PubMed Central, MedLine, CAS, SciSearch ${ }^{\circledR}$, Current Contents ${ }^{\circledR} /$ Clinical Medicine,

\section{Dovepress}

Journal Citation Reports/Science Edition, EMBase, Scopus and the Elsevier Bibliographic databases. The manuscript management system is completely online and includes a very quick and fair peer-review system, which is all easy to use. Visit http://www.dovepress.com/ testimonials.php to read real quotes from published authors. 